Itinéraires Itinéraires

Littérature, textes, cultures

2014-1 | 2015

Textualités numériques

\title{
Événement technodiscursif et sociabilités
}

\section{numériques}

Le cas de la « Révolte des 2.0 centimes » au Brésil en 2013

Rejane Arce

\section{OpenEdition}

\section{Journals}

Édition électronique

URL : http://journals.openedition.org/itineraires/2355

DOI : 10.4000/itineraires.2355

ISSN : 2427-920X

Éditeur

Pléiade

Référence électronique

Rejane Arce, «Événement technodiscursif et sociabilités numériques », Itinéraires [En ligne],

2014-1 | 2015, mis en ligne le 06 février 2015, consulté le 19 avril 2019. URL : http://

journals.openedition.org/itineraires/2355 ; DOI : 10.4000/itineraires.2355

Ce document a été généré automatiquement le 19 avril 2019

\section{cc) $(1) \ominus$}

Itinéraires est mis à disposition selon les termes de la licence Creative Commons Attribution - Pas d'Utilisation Commerciale - Pas de Modification 4.0 International. 


\section{Événement technodiscursif et sociabilités numériques}

Le cas de la « Révolte des 2.0 centimes » au Brésil en 2013

\section{Rejane Arce}

\section{Introduction}

\section{Pai, afasta de mim este cálice ${ }^{1}$. (Gilberto Gil et Chico Buarque, 1973)}

1 Pour tisser notre réflexion, nous nous inspirons de deux éléments fondamentaux : la distinction entre discours numérisé et discours numérique natif (Paveau 2013a), les notions d'événement énonciatif et d'événement discursif (Indursky 2005, 2002 ; Fenoglio 1997 ; Guilhaumou et Maldidier 1986a, 1986b), afin de comprendre quelques aspects des sociabilités (Casilli 2010) technolangagières de nos jours.

2 L'événement historique sur lequel s'appuie cet article est celui du mouvement protestataire, surnommé parfois «La révolte du vinaigre» ou «La révolte des 2.0 centimes", qui s'est déclenché au Brésil en juin 2013. L'événement d'énonciation (dorénavant énonciatif) plus précis concerne quatre ensembles discursifs publiés à ce propos le 18 juin 2013 sur les pages web de Radio France Internationale (RFI), du Nouvel Observateur, de Brasil de Fato et de Folha de São Paulo.

Les médias ont été choisis sur le critère des contenus en libre accès sur Internet, celui de leur ancienneté et de leur importance. On a essayé par ailleurs de respecter une certaine représentativité et diversité politique, au profit d'une approche rhizomatique et non dualiste, car il ne nous parait pas adéquat d'essayer de polariser les discours (en termes droite vs gauche).

4 Voici les quatre médias à partir desquels nous avons puisé nos exemples: RFI, qui diffuse depuis 1931, offre des informations en plusieurs langues et s'est spécialisé dans l'actualité internationale; Le Nouvel Observateur, né dans les années 1950, est un hebdomadaire très bien installé dans la presse française, s'inscrivant dans une filiation dite de gauche; Folha 
de São Paulo, fondé en 1921, est reconnu comme l'un des journaux les plus diffusés au Brésil (version papier et en ligne), préconisant une ligne éditoriale plurielle ; Brasil de Fato, enfin, est un journal créé au sein du Forum social mondial en 2003 par des mouvements sociaux.

Le travail se focalise sur les productions discursives suivantes: deux textes en français intitulés "Vaste mouvement de protestation au Brésil contre la vie chère ", sur $R \mathrm{FI}^{2}$ et "Le miracle économique perd de sa superbe ", dans Le Nouvel Observateur ${ }^{3}$; et deux autres en portugais intitulés « Em protesto de SP, maioria não tem partido, diz Datafolha ${ }^{4}$ » (« En manifestation à São Paulo, la majorité n'a pas de parti, dit Datafolha »), de Folha de São Paulo et «Luta contra tarifa incendeia o Brasil» (« La lutte contre les tarifs enflamme le Brésil ») de Brasil de Fato 5 .

Quand nous parlons de "texte", nous tenons compte d'une approche symétrique selon laquelle « l'analyse linguistique ne doit pas se contenter de porter sur des faits langagiers constitués uniquement de "langage" au sens traditionnel du terme, mais [...] la définition du langagier doit s'étendre à et s'enrichir de matérialités sociales, culturelles, environnementales, objectales, etc. » (Paveau 2013a : 3). Nous parlerons donc de «texte » à l'égard d'une unité imaginaire, d'un lieu de production de sens. Autrement dit, le texte est avant tout une "matérialité discursive ${ }^{6}$ ", et spécifiquement dans le cas des exemples traités ici, les matérialités sont multimodales, constituées à la fois par des éléments langagiers, des photos, des vidéos, des infographies, etc. D'autre part, nous ne traiterons pas non plus le texte et le discours comme des réalités susceptibles d'être distinguées. Par conséquent, nos considérations tiennent toujours compte d'une réalité discursive composite et indécomposable : on traite alors l'événement dans son irruption historique, par la mise en œuvre d'une structure, d'une mémoire et d'un événement.

Dans un premier temps, nous présenterons nos outils théoriques issus de l'analyse du discours franco-brésilienne, en particulier les notions d'événement énonciatif et d'événement discursif, en lien avec la notion de mémoire discursive. Nous proposerons ensuite une analyse de l'événement énonciatif que constitue le mouvement protestataire de juin 2013 au Brésil dans l'univers discursif numérique. Nous nous pencherons pour terminer sur des phénomènes qui articulent spécifiquement le technique et le langagier, et qui rendent les manifestations multi-sémiotiques en ligne irréductibles à une matérialité simplement verbale.

\section{1. Événement historique, énonciatif, discursif}

8 L'événement discursif «ne se confond ni avec le fait divers, ni avec le fait désigné par le pouvoir, ni même parfois avec l'événement construit par l'historien. Il est à saisir dans la consistance d'énoncés qui font réseau à un moment donné» (Guilhaumou et Maldidier 1986: 44, nous soulignons). Tout au long de ce travail, nous allons tenter d'approfondir cette notion dans le cadre des réseaux sociaux numériques (dorénavant RSN) en tentant de saisir a posteriori la consistance discursive de l'événement: il s'agit en effet d'une discursivité en cours, de laquelle nous ne pouvons guère analyser la configuration énonciative globale, encore inédite. 


\subsection{Inscription et circulation}

9 La notion d'événement, largement discutée en analyse du discours, sera abordée sous deux perspectives: nous considérerons à la fois que le fait, dans sa dimension pragmatique, crée l'événement, mais également qu'il existe une différence entre un événement énonciatif et un événement discursif, le passage entre les deux (ni obligatoire ni automatique) étant déterminé par des conditions de circulation et de diffusion des discours contemporains.

10 À notre sens, l'urgence de diffusion contemporaine ne permet pas le recul nécessaire pour analyser de façon approfondie les nuances des événements. Nonobstant, nous sommes loin de soutenir que la contemporanéité est un handicap pour la recherche ; au contraire, pour nous, elle est le lieu privilégié d'observation et d'analyse des nouvelles expressions de la subjectivité et des discours. En outre, quelle que soit la forme d'engagement ou de recul adoptée à l'égard d'une problématique de recherche contemporaine, nous vivons, nous agissons au sein de ces nouvelles expressions.

11 La nuance entre événement discursif et événement énonciatif sera établie à partir de la notion d'inscription dans un réseau de manière à contribuer à la formulation d'un certain discours social qui pourra, dans un ordre de succession, venir s'insérer dans la mémoire discursive ${ }^{7}$.

Dans cette perspective, nous prenons en compte la distinction établie par Courtine entre diffusion et circulation : «[...] la production d'une séquence discursive à partir d'une place au sein d'un appareil l'inscrit dans un réseau de diffusion des discours qui règle la circulation des formulations » (Courtine 1981:66, nous soulignons).

13 Ainsi, nous pouvons considérer Facebook (dorénavant $\mathrm{FB}$ ) comme un appareil inscrit dans les RSN, et cette inscription nous semble décisive, puisqu'elle gère la circulation. Autrement dit, il s'agit d'un réseau social collaboratif établi préalablement qui rend possible l'expansion, surtout à travers des commentaires et des partages, de discours provenant d'autres réseaux (des médias, qui transitent entre la version papier et la version digitale, et notamment des blogs, de Twitter, de YouTube, de TwitCasting, etc.) ; mais ce réseau peut également être la source propre des discours qu'il propage, comme les « événements » qui peuvent y être créés par les usagers.

14 Ajoutons que cette forme de discursivisation n'est pas occasionnelle, ce qui veut dire que les réseaux collaboratifs ne dépendent plus des médias "traditionnels" pour faire circuler un sujet. Par ailleurs, ils imposent un ordre différent de profusion des discours, puisqu'ils peuvent réunir toute sorte de matérialités. Les RSN sont en général multisémiotiques, présentant une navigabilité caractérisée par des segments, des mots, des images, des vidéos cliquables et dynamiques (ou pas).

Dans ce cadre, par exemple, et spécifiquement à l'occasion des manifestations de juin 2013 au Brésil, on a pu assister à l'éclosion d'un réseau de journalisme indépendant caractérisé par un contre-récit, c'est-à-dire une coupure avec le récit unifié et unificateur dicté par la dynamique économique ${ }^{8}$. Il s'agit du collectif "Mídia NINJA» (NINJA, acronyme portugais pour Récits Indépendants, Journalisme et Action) ${ }^{9}$.

16 Ce qui nous intéresse n'est pas le mouvement protestataire en soi, et il n'est pas non plus utilisé ici comme prétexte. Il fait l'objet de notre réflexion en vertu de la dimension globale que sa circulation a prise, une circulation qui a donné lieu à l'émergence des 
réseaux autour de la question, apparaissant surtout dans l'immédiateté. Dans cet univers numérique, nous prêtons attention aux formes de suivi d'un événement, autrement dit, nous nous intéressons aux formes technolangagières «dans lesquelles le langage est technique et la technique est langagière » (Paveau 2012a, 2013a), mises en évidence sous la forme de " gestes technolangagiers ${ }^{10}$ » qui recouvrent des formes d'engagement et de partage.

\subsection{Entre événement énonciatif et discursif : une transition}

17 Selon la lecture que nous faisons de quelques travaux d'Indursky (2002, 2005) - notamment inspirée des modalités de subjectivation proposées par Pêcheux (1975), soit l'identification, la contre-identification et la désidentification -, il existe une transition entre l'événement discursif et énonciatif ; bien qu'il ne soit pas toujours facile de distinguer l'un de l'autre, la différence entre eux est cruciale surtout en ce qui concerne l'appréhension de la singularité de l'événement.

Un événement énonciatif se caractérise par le fait qu'il rend notoire un changement dans un ordre discursif, bien qu'hétérogène. Ce changement peut être marqué par des glissements sémantiques, des déplacements d'un champ discursif vers un autre, des déplacements d'une position discursive vers une autre de nature contradictoire par rapport au domaine de mémoire de référence ${ }^{11}$. Ainsi, l'ensemble des dires de référence qui constituent une discursivité subit un mouvement qui provoque un changement dans la structure de cette mémoire, de façon à faire émerger une autre région ou stratification de cette même mémoire. Ce changement est régi par une perturbation qui s'instaure dans les relations d'alliance qui constituent un domaine de mémoire. Nous pouvons soutenir que dans un ensemble de dires possibles (formulations de référence qui se textualisent sous la forme de métaphores, reformulations, synonymies, etc.) une discordance émerge ; cependant, la relation métaphorique établie avec les formulations de référence est maintenue, bien que donnant lieu à un déplacement. De ce processus résulte un événement énonciatif, une division interne d'un domaine de mémoire.

En revanche, un événement discursif demande beaucoup plus qu'un changement dans le domaine de l'intradiscours. Ceci exige un changement intense dans l'ordre des dires au point d'instaurer une rupture. Cette rupture s'inscrit dans la rareté. Les événements discursifs, en ce sens, se produisent uniquement quand le "dissensus » (au sens de la mésentente de Rancière $1995^{12}$ ) a lieu. Dans un domaine de mémoire, un dire devient incompatible, étrange, car il provoque une rupture avec les formulations de référence de façon à configurer un nouveau domaine de mémoire, en vertu de cette dissension.

La distinction fondamentale entre les deux événements repose sur le fait que dans le premier cas, la différence est instaurée à l'intérieur d'une discursivité, inaugurant une branche, une nouvelle orientation discursive dans un domaine de mémoire. Dans le second cas, la différence est si décisive qu'elle crée son propre espace extérieur, une autre discursivité qui rompt avec son référent. L'événement discursif s'inscrit dans la rareté, il est lié à des ruptures historiques. Pour notre part, nous dirons encore que, même si une rupture est exigée, la relation avec un domaine de savoir et de mémoire sera toujours requise, car ce sera justement l'opposition, la rupture « par rapport à » qui constituera le guide de cet ordre discursif. En effet, les deux événements dépendent de "la nouveauté » liée à un événement historique. 
21 À l'ère du numérique, la relation entre événement discursif et énonciatif est mise en tension, à la fois par l'immédiateté et par les technodiscursivités, phénomènes qui demandent de nouveaux gestes de lecture. Dans ce contexte, les événements historiques s'avèrent des événements technodiscursifs en construisant des formes renouvelées de mémoire (numérique et discursive) et de partage des savoirs, surtout dans ce qui touche l'avènement des post-médias, soit l'appropriation du savoir-faire numérique par les sujets ordinaires. C'est l'un des aspects que l'on mettra en valeur concernant la construction discursive de l'événement qui fait l'objet de ce travail.

\section{L'événement historique et énonciatif dans l'univers discursif numérique}

\subsection{Spécificités discursives des univers numériques}

Précisons d'abord la notion d'« univers discursifs numériques ${ }^{13}$ » au moyen d'une typologie. En ce qui concerne les productions écrites, le terme numérique recouvre, selon Paveau, des réalités sémiotiques différentes :

- des discours imprimés numérisés pour l'analyse linguistique par exemple, donc non natifs mais qui acquièrent des traits natifs, par exemple la navigabilité ; constituant des bases de données, ils sont mis en ligne ou pas ; c'est le cas des corpus annotés des talistes par exemple ;

- des discours imprimés numérisés par scannage ou mise en ligne, dans un contexte éditorial, avec les mêmes propriétés acquises ; ils sont en ligne ; c'est par exemple le cas des revues numérisées sur le portail Persée ;

- des discours numériques natifs, produits directement en ligne, qui peuvent conserver la linéarité scripturale des écrits imprimés (pas de liens hypertexte, pas de réticularité, comme c'est souvent le cas sur les blogs littéraires par exemple) ou prendre des formes réticulaires grâce à l'hypertextualité (systématique et même automatique dans le cas des réseaux sociaux). (Paveau 2013a: 8, nous soulignons.)

Ainsi, les discursivités qui illustrent cette réflexion sont celles produites en ligne, donc relevant d'un discours numérique natif, comprenant, outre le texte, plusieurs formes technolangagières, en particulier des icônes, des boutons, des infographies, des vidéos, des galeries de photos, etc.

Par ailleurs, en ce qui concerne les formes de circulation et la nature de l'événement protestataire qui a eu lieu au Brésil en juin 2013, il nous semble assez proche de ce que Paveau (2011) a décrit à propos de la révolution tunisienne ou « Révolte de Sidi Bouzid » (2010-2011):

Il se trouve que nous vivons actuellement un événement remarquable qui est à la fois historique, discursif, politique, culturel, médiatique et bien d'autres choses encore. Cet événement a une caractéristique remarquable et remarquée par tous : sa dimension numérique ${ }^{14}$.

Dans ce cadre, ce qui nous intéresse particulièrement est la dimension numérique des manifestations au Brésil ; sa dimension politique restera encore à analyser dans un avenir proche où les conditions historiques seront peut-être moins nuageuses et les résultats discursifs de l'événement plus visibles. 


\subsection{Un événement énonciatif : la « révolution du vinaigre » ou "révolution des 2.0 centimes "}

La désignation «révolution des 2.0 centimes » vient du fait que la goutte qui a fait déborder le vase, en déclenchant des manifestations dans l'ensemble du pays, a été l'augmentation du tarif des transports en commun à São Paulo, la plus grande ville brésilienne et sa capitale économique, qui compte plus de onze millions d'habitants. Les 20 centimes écrits sous la forme 2.0 font référence à la génération $2.0 \mathrm{du}$ Web (le Web de la simplicité, de l'interactivité et de ladite intelligence collective ${ }^{15}$ ), plus particulièrement celle qui est « sortie de Facebook », slogan circulant amplement lors des manifestations et sur lequel nous reviendrons.

La suite des événements a démontré que les manifestations avaient comme objet toute une série de revendications à la fois précises et génériques, qui mettaient surtout en cause les dépenses publiques pharaoniques au profit de la Coupe du monde de football prévue au Brésil en 2014, au détriment d'investissements dans les domaines de la santé, de l'éducation et des infrastructures publiques.

En outre, la révolution des 2.0 centimes renvoie au fait que les manifestants étaient pour la plupart des jeunes ${ }^{16}$ qui portaient des pancartes sur lesquelles l'énoncé « Nous sommes sortis de Facebook » était apposé. Par conséquent, plusieurs médias leur ont attribué le rôle de protagonistes de l'événement ${ }^{17}$.

En effet, un énoncé circulant amplement dans ce contexte, sur les pancartes et dans les médias, intègre les significations de cette désignation : «Ce n'est pas pour 20 centimes. » L'énoncé « original » et métaphorisé a fait l'objet de plusieurs productions numériques, particulièrement des photomontages avec des personnalités du monde teen ${ }^{18}$.

Un autre exemple de métaphorisation inspirée du mouvement protestataire est une chanson diffusée le 21 juin 2013 sur YouTube. Le compositeur et chanteur Thiago Correa l'a créée à partir des énoncés inscrits sur les pancartes et banderoles utilisées lors des manifestations. Intitulée, Brasil em cartaz (Brésil à l'affiche) ${ }^{19}$, elle a totalisé plus de 90000 vues en dix jours ${ }^{20}$. Le compositeur, adepte du style musical mashup ${ }^{21}$ qu'il fait circuler sur sa chaîne YouTube (lieu principal de diffusion de son travail, en accès libre et gratuit), a écrit la chanson en faisant un montage constitué d'énoncés populaires métaphorisés, notamment certains de ceux figurant sur les pancartes et banderoles, en plus d'extraits de chansons célèbres réinterprétées. La chanson a pour refrain l'énoncé «Saí do Facebook para mostrar como se faz " ( Je suis sorti de Facebook pour montrer comment faire »).

En effet, la fronde a donné lieu à une prolifération de photomontages présentant des personnalités tenant des pancartes, banderoles ou affiches en faveur du mouvement, en particulier des créations $^{22}$ témoignant des nombreuses possibilités de relecture et d'appropriation des discursivités circulantes grâce aux RSN. Il nous paraît important de souligner qu'il devient fréquemment impossible de récupérer l'origine d'une matérialité de cette nature. D'ailleurs cette impossibilité est également utilisée comme une stratégie pour éviter de laisser des traces en cas de polémique. Une manœuvre utilisée par de nombreux utilisateurs de FB est en effet de couper et coller des informations et non pas de les partager (ce qui impliquerait de maintenir l'origine et par conséquent la traçabilité). Cela constitue une tentative de défense contre la " persécution », la censure en ligne. 
31 En outre, une page concernant la fronde a été créée sur l'encyclopédie collaborative Wikipedia, intitulée «Mouvement protestataire de 2013 au Brésil ${ }^{23}$ " sous réserve, annoncée sur la page, qu'il s'agissait d'un «événement en cours », rendant la page provisoire $^{24}$. Dans le but de maintenir une petite archive concernant la question, nous avons créé deux « histoires » sur Storify ${ }^{25}$ (extraits en captures d'écran présentés dans les figures 1 et 2, ci-dessous), mettant en avant les différentes désignations attribuées à l'événement protestataire et son processus de métaphorisation, soit d'appropriation/ relecture des dires par les sujets.

Fig. 1. Extrait du Storify « La révolte du vinaigre»

(9) Storify by Rejane Arce 1 ys 4 minutes

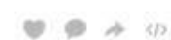

La révolte du vinaigre

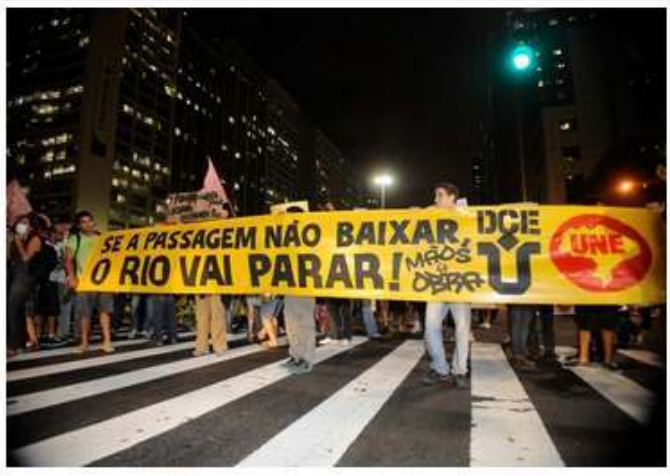

File:Protestos no Rio em 2013.jpg (3) WIKMEDLA ILYASMINUTES

Mouvement protestataire de 2013 au Brèsil

Le Mouvement protestataire de 2013 au Brésil est une série de

manifestations de masse qui touchent le Brésil a partir de mars 2013.

lles.

http://storify.com/rarce/revolte-du-vinaigre 


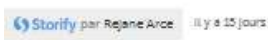

la révolution des 2.0 centimes

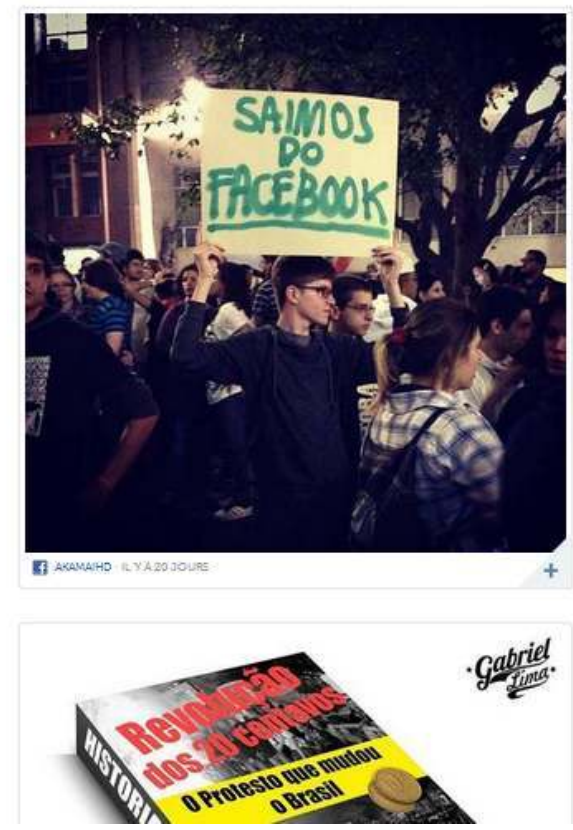

https://storify.com/rarce/la-revolution-des-2-0-centimes

Les discursivités présentées dans nos deux recueils Storify illustrent le caractère technodiscursif du mouvement, en plus des formes d'appropriation et de transformation des discours. La désignation " Révolution du vinaigre ", par exemple, vient du fait que les manifestants utilisaient un morceau de tissu imprégné de vinaigre pour supporter les effets du gaz lacrymogène lancé contre eux par la police lors des manifestations ; dans ce contexte, porter du vinaigre sur soi pouvait être interprété comme un crime passible d'emprisonnement ${ }^{26}$.

Ces formes d'historicisation d'un événement témoignent de la délinéarisation du récit du temps présent, surtout quand il s'agit d'«organiser " un ensemble de matérialités technolangagières, comme le montre bien un récit de l'événement publié sur un blog ${ }^{27}$. Il est construit à partir de vidéos de YouTube, de galeries de photos, de citations littéraires, d'une chanson populaire brésilienne (MPB), d'extraits de pages $\mathrm{FB}$, de références à des pages d'information ainsi qu'à la page du mouvement qui a convoqué, à São Paulo, les premières manifestations (Mouvement passe libre, en portugais, Movimento Passe Libre $\mathrm{MPL}^{28}$ ), à des blogs, etc. Tout cela ne constitue pas simplement des redirections vers un " extérieur » et ces éléments ne sont pas non plus des ornements illustratifs de ce qui est langagier. Ces bribes et montages constituent le texte en lui-même, où le technique et le langagier sont métissés et incontournablement constitutifs des sens des formes technolangagières et discursives. C'est pourquoi on ne peut pas proposer une analyse compartimentée : la langue, le discours et la technique sont à part entière dans le processus de discursivisation. 


\section{Formes technolangagières d'engagement}

Fig. 3. Exemples de boutons
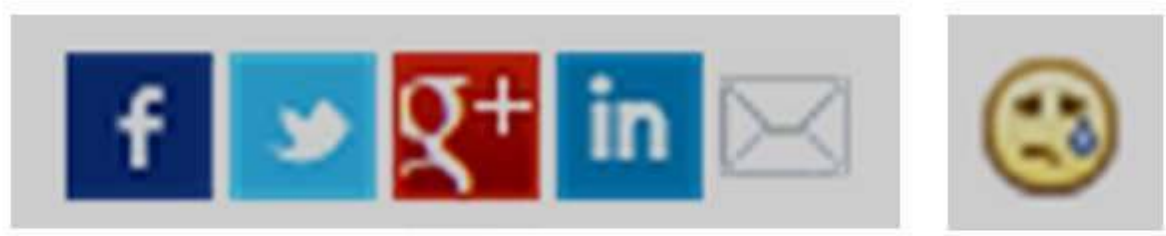

\section{+1 Ajouter}

Nous avons mentionné plus haut une distinction entre discours numérisés et numériques natifs formulée par Paveau. Au-delà d'une préoccupation dénominative, établir cette distinction nous semble fondamental pour souligner le caractère intraduisible des formes technolangagières et technodiscursives, c'est-à-dire l'impossibilité de conversion de ce qui est produit dans les environnements numériques en formes « purement» langagières. À ce propos, nous adoptons la pensée de Paveau (2012a) quand elle attribue aux «boutons » (avec ou sans texte en pop-up), que nous avons mis en illustration ci-dessus, un fonctionnement discursif qui recouvre toute une série d'actions et réactions vers un objet ou un sujet, de façon à établir des sociabilités numériques.

\section{1. «On ne peut pas parler dans la vie comme on parle par texto ou sur Facebook »}

À ce propos, nous voudrions citer un extrait d'un spectacle de Gad Elmaleh disponible sur YouTube $^{29}$, qui exemplifie d'une façon assez anecdotique et humoristique, l'impossibilité d'un passage sans perte de sens du technodiscursif à une forme de la langue. Le comédien présente en effet plusieurs situations qui illustrent le fait que «l'on ne peut pas parler dans la vie comme on parle par texto ou sur Facebook ». C'est le cas des smileys : celui qui présente quelqu'un qui pleure, par exemple, comment pourrait-on le « traduire »?

Une personne qui reçoit un non comme réponse à une invitation ne pourrait pas traduire sa déception comme il est possible de le faire par l'utilisation de l'émoticône ci-dessus. De même, le comédien joue une situation où quelqu'un entre dans un restaurant, s'adresse immédiatement à un inconnu et lui fait une demande d'amitié " comme sur FB", soit par le biais du bouton « Ajouter ", en utilisant toutefois les « outils » du langage verbal, c'està-dire en posant directement la question: "Tu veux être mon ami?» La réponse probable serait : «Non, je t'ignore ${ }^{30}$. » Cela montre que ces formes technodiscursives sont des schémas relationnels, des liaisons numériques et surtout des genres discursifs intraduisibles (Paveau 2013a) ${ }^{31}$. 

différence établie par Orlandi (2010) entre série et réseau. Selon elle, la «mémoire métallique $^{32}$ " produite par les médias grâce à l'utilisation des nouvelles technologies n'a pas d'historicité sinon celle de la machine. Elle est ainsi horizontale et non pas verticale, et produite en série, sous la forme de l'addition, de l'accumulation: ce qui est dit ici s'ajoute à ce qui est dit ailleurs en produisant un "effet de réseau». La mémoire métallique produit la quantité et non l'historicité.

Nous sommes d'accord avec Orlandi sur la question de la quantité qui produit l'effet de réseau sérialisé ; cependant, il faut considérer que le Web 2.0 facilite la production de l'historicité. À l'aide d'un ordinateur en effet (tablette, smartphone, tout dispositif connecté à Internet), on produit de l'historicité, de la mémoire discursive, dans un processus à la fois technique et subjectif, en métaphorisant la quantité. C'est ce que nous avons tenté d'illustrer au travers des exemples circulant lors des manifestations (la chanson, les énoncés réinterprétés, les montages photographiques redocumentés sur Storify, etc.), notamment sur les pancartes, banderoles et sous la forme de récit multimodal ${ }^{33}$. Cela ne se passe pas toujours sans contrainte et la quantité (l'effet de série) peut s'avérer nuisible dans le processus de lecture. Malgré cela, sur les réseaux, des matérialités de l'histoire, du cinéma, des médias, provenant des différents domaines de la connaissance et sous différents registres discursifs et supports, produisent des échos, s'entrecroisent, s'affrontent, se complètent, se réfutent et ouvrent des univers de compréhension et d'analyse.

\subsection{Discursivités partagées}

captures d'écran ci-dessous ont pour but d'illustrer ce que nous avons tenté de soutenir jusqu'ici, c'est-à-dire le caractère intraduisible des formes de partage technodiscursives. Dans l'ensemble, les articles soulignent fortement les chiffres du mouvement protestataire: le nombre de manifestants, de blessés, d'arrêtés; les dommages au patrimoine public, la violence, le chaos, etc. Autrement dit, ils offrent une vision panoramique, voire superficielle, des premiers jours de l'événement.

Il est intéressant de remarquer que la multimodalité fonctionne autrement dans les médias repérés pour illustrer notre réflexion. Tandis que dans les RSN elle est construite en quelque sorte sur le principe dialogique, en apportant de multiples voix aux discours, dans nos exemples, la référentialité est établie de façon interne : les sources proviennent, pour la plupart, d'un réseau professionnel dans lequel il n'y a quasiment pas de place pour les voix citoyennes.

41 Les formes de partage ou de recommandation des articles sont plus remarquables que les formes de commentaire, le genre du commentaire restant une forme de discours contrôlée par le détenteur de la discursivité source (il faut s'identifier, se connecter sur un compte existant lié à un réseau ou créer un compte dédié à ce propos). Le texte issu de RFI ne présente par exemple aucun commentaire contre 101 partages sur FB le 22 juin 2013 :

\section{RFI}

Texte : « Vaste mouvement de protestation au Brésil contre la vie chère » [BRÉSIL Article publié le mardi 18 juin 2013 à 09 h 01 - Dernière modification le : mardi 
18 juin 2013 à 10 h 35], http://www.rfi.fr/ameriques/20130618-vaste-mouvementprotestation-bresil.

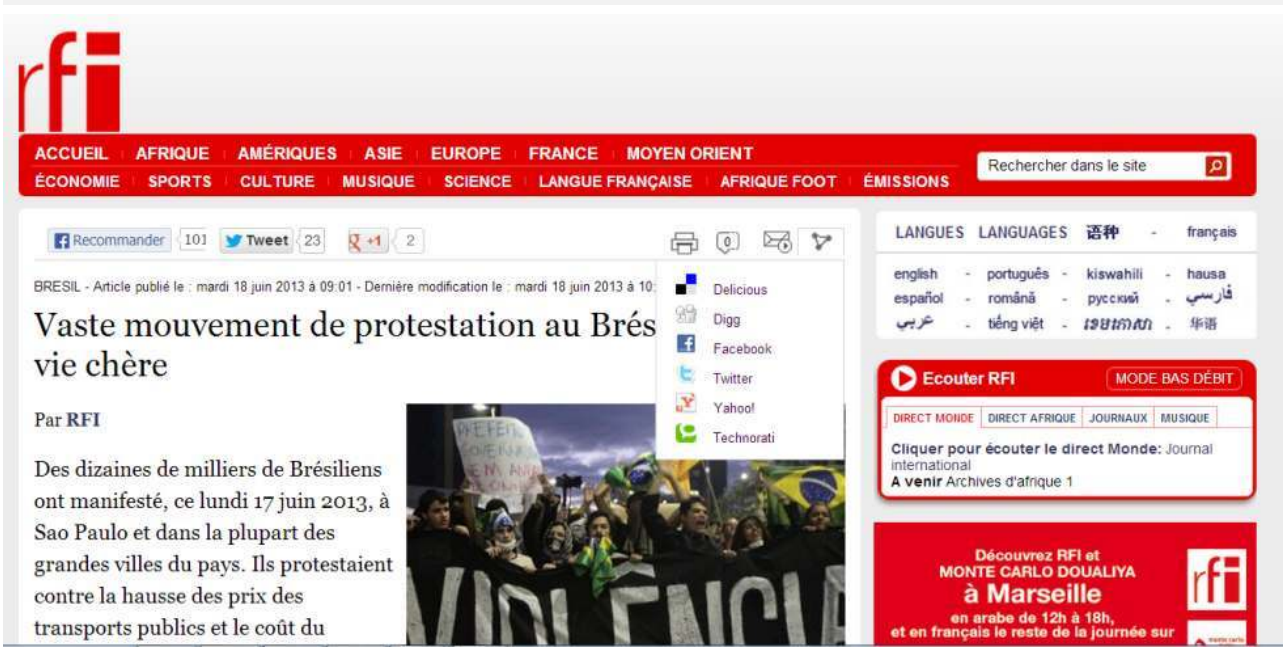

Le 22 juin à $14 \mathrm{~h} 50$ le texte présentait 0 commentaire ; pour réagir, il faut avoir un compte RFI, FB ou Twitter ; il est possible de partager le document si l'on se connecte sur FB, Twitter ou Google+1, etc.

Le 22 juin à $14 \mathrm{~h} 51$, le texte présentait 101 partages sur FB, 23 sur Twitter et 2 sur Google +1 .

Le partage ouvre la possibilité d'un lien plus «flou » et par cela nous ne voulons pas signifier faible. Le partage dans un espace « plus ou moins » personnel ou personnalisable comme FB (vers lequel la plupart des partages sont orientés, en fonction, on suppose, de la facilité d'utilisation) est aussi une manière de s'écarter du contrôle juridique que les médias imposent aux commentaires.

D'un autre côté, les 11 commentaires déclenchés à partir de l'article du Nouvel Observateur (capture d'écran ci-dessous) par exemple, sont des réactions à un contexte plus large que celui présenté dans le texte, et sont notamment critiques (voir par exemple les commentaires de Diogène d'Arc et Renata Lopes Araujo ${ }^{34}$ ).

\section{LE NOUVEL OBSERVATEUR}

Texte : BRÉSIL. Le miracle économique perd de sa superbe [Créé le 18 juin 2013 à $11 \mathrm{~h} 09$ - Mis à jour à $14 \mathrm{~h} 40$ ] http://tempsreel.nouvelobs.com/economie/20130618.OBS3696/bresil-le-miracleeconomique-perd-de-sa-superbe.html. 


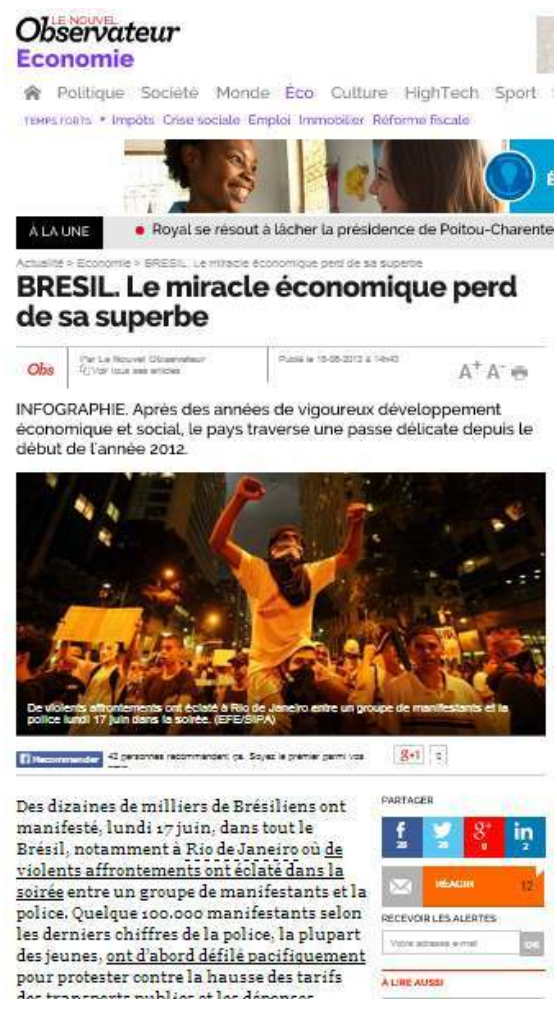

Le 22 juin à 14 h 56 le texte présente 38 partages (recommandations) sur FB. Le site offre des possibilités de partages sur FB, Twitter, Google+, LinkedIn et par e-mail. Le 22 juin à $14 \mathrm{~h} 57$ le document présentait 11 commentaires ou réactions. Pour commenter, il faut se connecter sur FB, Twitter, Google +, Yahoo ou LinkedIn ou s'inscrire sur RFI (capture d'écran ci-dessous).

\section{Je me connecte}

\begin{tabular}{|l|l|}
\hline $\mathbf{f}$ & $\begin{array}{l}\text { avec mon compte } \\
\text { Facebook }\end{array}$ \\
\hline $\mathbf{S}$ & $\begin{array}{l}\text { avec mon comple } \\
\text { Twitter }\end{array}$ \\
\hline $\mathbf{8 +}$ & $\begin{array}{l}\text { avec mon compte } \\
\text { Googlet }\end{array}$ \\
\hline $\mathbf{Y t}$ & $\begin{array}{l}\text { avec mon compte } \\
\text { Yahoo! }\end{array}$ \\
\hline in & $\begin{array}{l}\text { avec mon compte } \\
\text { Linkedin }\end{array}$ \\
\hline
\end{tabular}

\section{Le Plus}

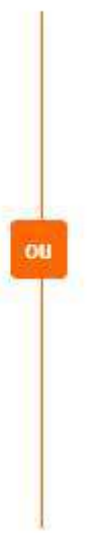

Mon email

Mon mot de passe

Mon mot de passe

Valider

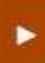

J'ai oublié mon mot de passe
Mon email 

trouvent des possibilités renouvelées de se faire écouter, de réagir et aussi de se rassembler, comme l'explique Castells :

Obviamente, la tecnología no determina ningún movimiento ni ningún comportamiento social. Pero Internet y las redes de telefonía móvil no son simples herramientas, sino formas de organización, expressions culturales y plataformas específicas de autonomía política ${ }^{35}$. (Castells 2012 : 108)

Par ailleurs, selon l'analyse de Castells portant sur l'avènement des post-médias, les univers numérique et urbain ${ }^{36}$ forment par assemblage un troisième espace, qu'il désigne comme espace de l'autonomie : «Este híbrido de ciberespacio y espacio urbano constituye un tercer espacio que yo llamo el espacio de la autonomia ${ }^{37}{ }$ (Castells $2012: 213$ ).

On notera à ce propos les extraits illustrant la configuration numérique de l'événement protestataire, relativement au rôle du réseau $\mathrm{FB}$ :

[...] ao menos em São Paulo (SP), pela primeira vez o número de confirmados em um evento marcado pelo Facebook praticamente se equiparou com o número de pessoas nas ruas. Das 285 mil que haviam dito que participariam do Quinto Ato contra o aumento das passagens, 250 mil pessoas tomaram as ruas. (BDF, « Luta contra tarifa incendeia o Brasil », 17 juin 2013. Nous soulignons $\left.{ }^{38}\right)$.

Traduction ${ }^{39}$ :

[...] au moins à São Paulo (SP), pour la première fois le nombre de participants confirmés à un événement créé sur Facebook a été pratiquement équivalent au nombre de personnes dans les rues. Parmi les 285000 personnes qui avaient dit qu'ils participeraient au $5^{\mathrm{e}}$ acte contre l'augmentation du prix des tickets de bus, 250000 personnes sont descendues dans les rues.

A maioria tem entre 26 e 35 anos e $81 \%$ se informaram do ato pelo Facebook. No total, $85 \%$ dos presentes buscaram informações pela internet. (FSP, «Em protesto de SP, maioria não tem partido, diz Datafolha », 18 juin 2013. Nous soulignons ${ }^{40}$ ).

Traduction:

La plupart [des manifestants] ont entre 26 et 35 ans et $81 \%$ se sont renseignés sur la manifestation via Facebook. Au total, $85 \%$ des présents ont cherché des informations sur Internet.

Pour revenir sur la question des partages, nous dirons que les formes de partage ouvertes par les médias plus ou moins «traditionnels » portant sur un sujet polémique trouvent sur les RSN un changement de voie/voix, une appropriation technodiscursive qui sert à fonder d'autres espaces d'expression. Cela témoigne aussi du fait que les médias privilégiant une certaine linéarité narrative ne sont plus les sources majeures d'information. Pour illustrer ce point, nous détaillons quelques aspects structurels et argumentatifs des discursivités en discussion.

D'un point de vue structurel, les quatre documents sont constitués de la manière suivante :

- RFI : texte + 1 photo (Reuteurs/Alex Almeida);

- Le Nouvel Observateur (dorénavant NO) : texte + 1 photo (EFE/SIPA) + infographie (Le Nouvel Observateur/Banque mondiale) + galerie de 19 images (Tasso Marcelo/AFP) + une vidéo de France 24 ;

- Brasil de Fato (dorénavant BDF) : texte + 1 photo (Mídia Ninja) + galerie Flickr de 23 photos (brasildefato1) + vidéo YouTube (TV Folha, modifié) ;

- Folha de S. Paulo (dorénavant FSP) : texte + galerie de 30 photos (Eduardo Anizelli/Folhapress) + 4 infographies (Editoria de Arte/Folhapress).

Itinéraires, 2014-1 | 2015 

brésiliens tentent de mettre en avant les protagonistes des manifestations par le biais des chiffres et des lignes politiques, tandis que les deux médias français s'efforcent de présenter les raisons économiques (à l'exemple de l'infographie du NO, avec les chiffres de l'inflation, du chômage, de la croissance et de la pauvretée ${ }^{41}$, et du titre de RFI), en plus des chiffres des manifestations et de l'évocation de la crainte de l'insécurité devant la Coupe du monde de football.

D'ailleurs, la question de la sécurité et des affrontements avec la police est présente dans les quatre discursivités, en particulier à travers une caractérisation dualiste de la manifestation (violente $v s$ non violente), de façon toutefois assez différente dans les cas de BDF (voir les segments en italique) :

RFI, « Vaste mouvement de protestation contre la vie chère " (18 juin 2013) ${ }^{42}$ :

À São Paulo, une manifestation pacifique a réuni plus 65000 manifestants sans heurts à déplorer avec la police alors que la semaine dernière des affrontements avaient laissé de très nombreux blessés sur le trottoir. (Nous soulignons.)

NO, BRÉSIL, « Le miracle économique perd de sa superbe » (18 juin 2013) ${ }^{43}$ :

Des dizaines de milliers de Brésiliens ont manifesté, lundi 17 juin, dans tout le Brésil, notamment à Rio de Janeiro où de violents affrontements ont éclaté dans la soirée entre un groupe de manifestants et la police. Quelque 100000 manifestants selon les derniers chiffres de la police, la plupart des jeunes, ont d'abord défilé pacifiquement pour protester contre la hausse des tarifs des transports publics et les dépenses excessives engagées pour l'organisation du Mondial 2014 de football. (Les hyperliens sont de NO. Nous soulignons.)

BDF, « Luta contra tarifa incendeia o Brasil » (17 juin 2013) $)^{44}$ :

Inicialmente, a mídia condenou veementemente as manifestações, classificou os jovens como vândalos e ressaltou a presença de uma classe média sem motivos para revolta. Alguns portadores de cartazes, já percebendo essa manobra de transformar um protesto legítimo em uma ação despolitizante "contra a corrupção», de luta "por direitos» e de que finalmente o Brasil «acordou», traziam consigo frases como «Se você acordou agora, saiba que a periferia nunca dormiu» ou "Saiba que já têm brasileiros acordados há tempos, você que não dava bola. [...]

Aos gritos de «Que coincidência, sem a polícia não tem violência»... (Nous soulignons.)

Traduction - BDF, «La lutte contre les tarifs incendie le Brésil» (17 juin 2013) :

Initialement, les médias ont fermement condamné les manifestations, ont qualifié les jeunes de vandales et ont souligné la présence d'une classe moyenne sans aucune raison de se révolter. Certains qui tenaient des pancartes, se sont rendu compte de cette manœuvre pour transformer une protestation légitime en action de dépolitisation " contre la corruption », de lutte pour «des droits ", et évoquant «le réveil » du Brésil, ils présentaient des slogans comme « Si vous vous êtes réveillés maintenant, sachez que la périphérie n'a jamais dormi » ou «Sachez qu'il y a des Brésiliens réveillés depuis longtemps, c'est vous qui vous en fichiez ». [...] Aux cris, "Quelle coïncidence, sans la police il n'y a pas de violence »...

FSP, «Em protesto de SP, maioria não tem partido, diz Datafolha " (18 juin 2013) ${ }^{45}$ :

Gritando frases como «O povo unido não precisa de partido» e «Sem partido, sem partido» os manifestantes se reuniram de forma pacífica. [...]

Durante o percurso, os manifestantes pediam para que as bandeiras de partidos políticos fossem guardadas. (Nous soulignons.)

Traduction - FSP, « En manifestation à São Paulo, la majorité n'a pas de parti, dit Datafolha " (18 juin 2013) :

En criant des phrases comme «Le peuple uni n'a pas besoin de parti » et «Sans parti, 
sans parti » les manifestants se sont réunis de manière pacifique. [...]

Durant le parcours, les manifestants ont demandé que les drapeaux des partis soient rangés.

51 À notre sens, le partage établit une liaison tissée par un ensemble de demandes formulées de façon subtile et "intraduisible", correspondant à des schémas technodiscursifs (englobant des aspects linguistiques, sociaux, idéologiques et techniques partagés) : demande de lire, voir, écouter et venir avec l'autre. Il ne s'agit pas d'une économie linguistique, il ne s'agit pas non plus d'un outil qui remplace les « mots » ou une longue explication. Il s'agit d'un espace technorelationnel (Paveau 2013b) de liaisons qui s'établissent comme un genre de discours (Paveau 2013a). Cela souligne encore une fois le rôle des RSN dans le cadre de la formulation de différentes pratiques au sein des univers numériques :

Un média social est donc, sur le plan discursif, un dispositif de discours constamment en évolution qui repose sur les relations entre les agents de la production verbale : la lecture et l'écriture en ligne sont orientées par les lectures et écritures des autres membres du réseau. Sur un RSN, la production discursive possède des caractéristiques fortes qui impliquent la nécessité d'une approche écologique portant sur l'ensemble du dispositif de production verbale et non sur un seul de ses éléments, comme l'énoncé ou le locuteur. (Paveau 2013b : 4)

Pour conclure la question des partages et des commentaires, nous remarquons que d'un point de vue quantitatif, ils sont beaucoup plus expressifs sur la FSP, soulignant en quelque sorte la représentativité de ce journal au Brésil en fonction de sa large circulation $^{46}$.

\section{FOLHA DE SÃO PAULO}

Texte : « Em protesto de SP, maioria não tem partido, diz Datafolha » [18 juin 2013 - $05 \mathrm{~h} \mathrm{00]}$

http://www1.folha.uol.com.br/cotidiano/2013/06/1296886-em-protesto-de-spmaioria-nao-tem-partido-diz-datafolha.shtml.

\section{cotidiano}

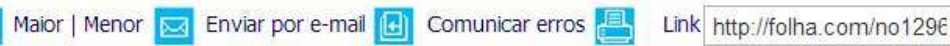

\section{8/06/2013 - 05h00 \\ Em protesto de SP, maioria não tem diz Datafolha}

DE SÃO PAULO

\footnotetext{
If Recomendar $2,8 \mathrm { mil } \quad \overline { Q } + 1 \longdiv { 1 1 4 }$
}

Em São Paulo, 65 mil pessoas se reuniram no largo da Batata, zona oeste, para participar do protesto contra o aumento da tarifa do transporte. Desses, $84 \%$ não têm preferência partidária, de acordo com o Datafolha.

Gritando frases como "O povo unido não precisa de partido" e "Sem partido, sem partido" os manifestantes se reuniram de forma pacífica.

Ao contrário do protesto anterior, na quinta-feira, em que houve mais de 200 feridos -

Le texte de la FSP peut être partagé sur Twitter, FB, Orkut, Google, Windows Live, etc. 
Le 18 juin à 5 heures ${ }^{47}, 2800$ personnes avaient partagé le texte sur FB et 114 sur Google+1.

Le 22 juin à 14 h 49, le texte comptait 99 commentaires (le dernier commentaire datant du 18 juin à $09 \mathrm{~h}$ 32). Seuls les abonnés au journal peuvent publier des commentaires de façon illimitée ; ceux qui sont simplement enregistrés sur le site peuvent commenter, mais ces commentaires sont filtrés et 20 seulement seront mis en ligne ${ }^{48}$

Page des commentaires : http://comentarios1.folha.com.br/comentarios/5902490? skin=folhaonline.

\section{目 COMENTE VEJA OUTROS TEXTOS PARA VISITANTES COMENTAREM}

\section{+ LIVRARIA}

- 'Estive hoje em um campo de concentração nazista'

- Freud examina o que mantém uma multidão coesa

- 'Holocausto Brasileiro' resgata histó ria de 60 mil mortos em hospício mineiro

- Marquesa de Santos fazia jogos sexuais com d. Pedro 10; ouca pesquisador

- Esquerda é incapaz de propor saidas para a crise atual, diz marxista

Comentar esta reportagem

Mordechai Ben Zion (8519)
Ver todos os comentários (99)

(18/06/2013 09h32) há 3 dias

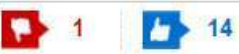

Os partidos politicos são FUNDAMENTAIS, NECESSÁRIOS E INDISPENSÁVEIS, para o correto funcionamento de uma democracia, são eles que dão foco e canalizam os anseios, aspirações e necessidades da população. Porém, não existem partidos no Brasil desde há muito tempo, o que existe são "condominios fechados" dedicados a obter vantagens para seus "poucos condôminos" garantir essas vantagens pelo maior periodo de tempo possivel. Como resolver isso ?? Pergunta muito complicada, não sei.

O comentário não representa a opinião do jornal; a responsabilidade é do autor da mensagem

D'autre part, malgré la nécessité de s'identifier à travers un compte pour pouvoir commenter un sujet, il apparaît que l'anonymat n'est pas non plus la meilleure solution, comme le montre le nombre de commentaires peu expressifs de BDF ci-dessous.

\section{BRASIL DE FATO}

Texte : « Luta contra tarifa incendeia o Brasil

[17 juin 2013]

http://www.brasildefato.com.br/node/13263. 


\section{BRASIL DE FATO}

Sábado, 22 de junho de 2013 Buscar

BRASIL. MUNDO ENTREVISTA CULTURA EDTTORIAL OPINIÃO CHARGES VIDEOS CURSOS

Ińcio - Luta contra tarifa incendeia o Brasil

\section{Luta contra tarifa incendeia o Brasil}

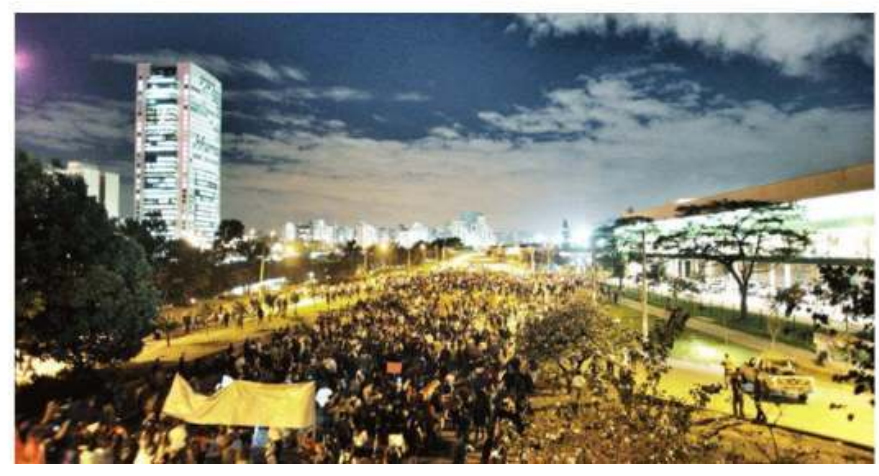

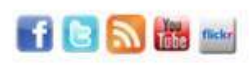

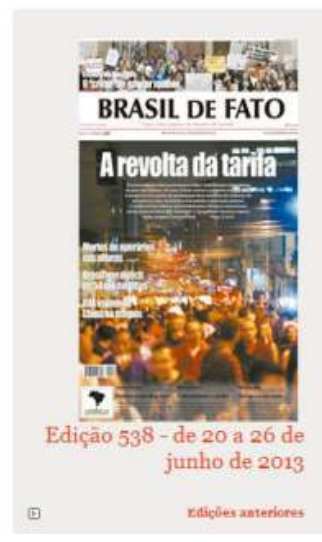

Le 22 juin à 15 heures, le texte présente 5 commentaires publiés entre le 18-06-2013 à $9 \mathrm{~h} 38$ et 19-06-2013 à $10 \mathrm{~h}$ 48. Les formes de partage possibles sont FB, Twitter, Orkut, e-mail. Le nombre de partages n'est pas indiqué.

Le site offre la possibilité de commenter anonymement, ce qui apparemment n'encourage pas plus les lecteurs (voir capture d'écran ci-dessous), vu la participation assez timide, sans vraiment instaurer d'interaction entre les participants, en comparaison de ce qu'on peut constater dans les commentaires de la FSP et du NO (par l'interpellation directe d'ailleurs, dans le dernier cas), par exemple, où les participants en effet réagissent, souvent, en fonction des autres interventions. 


\section{DEIXE SEU COMENTÁRIO}

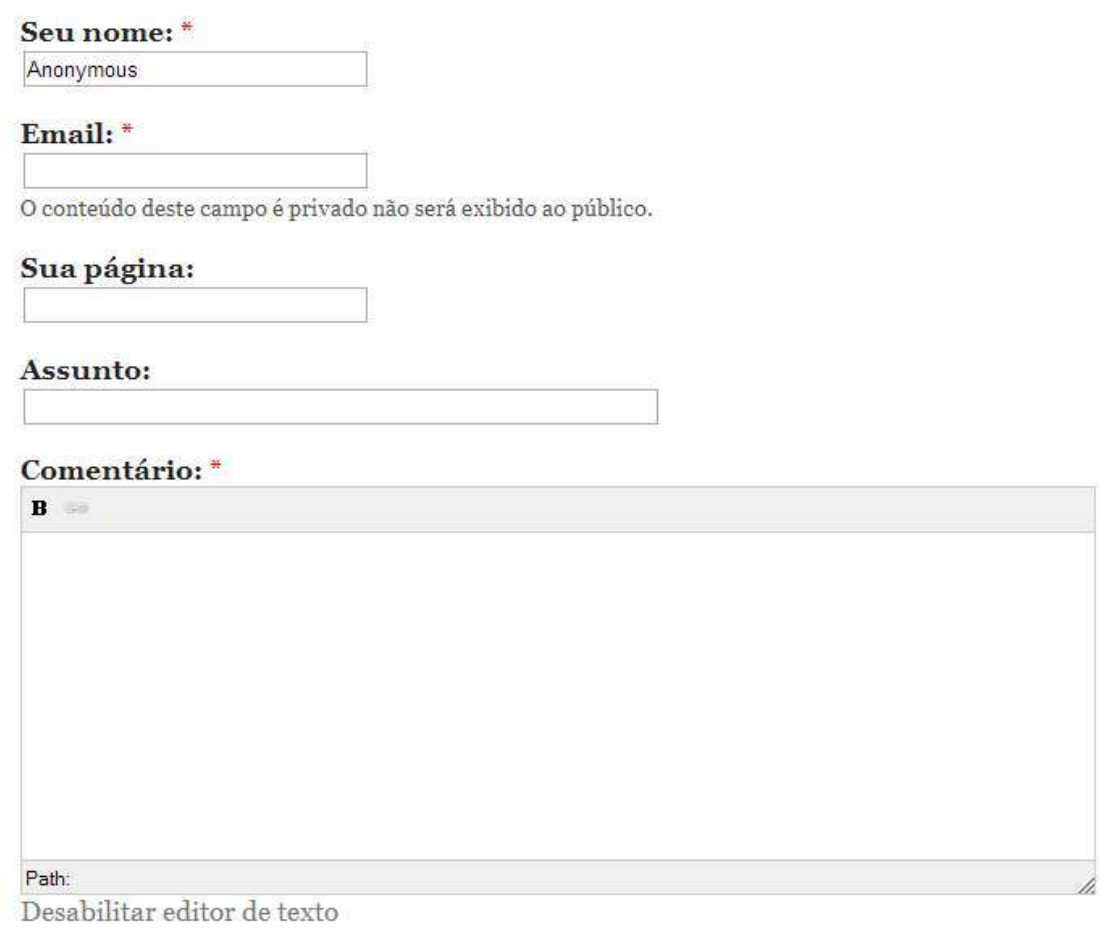

\section{Conclusion}

Les discursivités des RSN constituent ce que Pêcheux a nommé «circulationaffrontement » ([1983] 1990 : 307) ${ }^{49}$, c'est-à-dire un réseau de contre-discours par rapport aux formes de raconter / informer / mettre en discours des médias, dans la mesure où ces discursivités construisent, à propos d'un "même" référent, d'autres significations, pleines de bribes, de voix autres, souvent dissonantes. Ces significations produisent, par conséquent, un effet de contre-série (puisqu'il n'y a pas de relation paraphrastique entre les textualités) et de fondation de nouveaux réseaux via l'appropriation, la coupure du flux de dires des réseaux conventionnels.

C'est le cas des discursivités que nous avons documentées dans nos deux recueils Storify et mentionnées au cours de ce texte: d'une part "La "révolte du vinaigre" secoue le Brésil ${ }^{50}$ ", publiée sur le site web Global Voices, construite surtout à partir de vidéos YouTube du collectif Anonymous Brasil, vidéos de gens ordinaires, de collectifs, de segments cliquables, de tweets, récits, etc.; d'autre part le récit Storify «Au Brésil, les manifestations contre la vie chère tournent au vinaigre $»^{51}$, de Thomas Bourdeau, composé de hashtags, vidéos YouTube, tweets, contenus de pages FB, versions en anglais de l'événement, ainsi que « Pour tout savoir sur les événements au Brésil, rendez-vous sur les réseaux sociaux...", du même auteur, lequel traite le caractère technodiscursif de l'événement en utilisant à part entière cette forme d'expression numérique ${ }^{52}$. Dans ce cadre, nous pouvons encore nous référer au billet « Le Brésil a déjà gagné ${ }^{53}$ ", du blog de Joanna Espinosa, sur le site de Mediapart, le 18 juin 2013, construit, comme nous l'avons évoqué, à partir d'une multitude d'éléments multi-sémiotiques.

Ces textualités, en tant que formes de circulation-affrontement par rapport aux médias «traditionnels», aident à produire l'événement technodiscursif (ni énonciatif, ni 
discursif), constituant de ce fait un phénomène de caractère nouveau, par le biais des post-médias (au sens de Castells 2012), qui produisent à la fois une mémoire et un événement technodiscursifs. Les outils et les formes technolangagières sont là pour nous aider à participer à l'histoire du temps présent, cette histoire qui nous appartient. Ceci peut commencer par l'acte assez banal de partager une information, de façon à lui donner des directions rhizomatiques aux contours imprévus, face à la dimension subjective que le flux des discours acquiert en vertu de l'appropriation technodiscursive et technopolitique du Web 2.0 par les sujets. Les liaisons technolangagières, la sociabilité ancrée sur les partages d'un événement, contribuent à la création et à l'historicisation de l'événement. Cette possibilité se trouve augmentée par les RSN, dans la mesure où il existe un continuum entre univers urbain et numérique, constituant un lieu hybride de production de discours et surtout de contre-discours. Il se produit alors une restructuration du travail de lecture et de production/profusion de dires, puisque les sources privilégiées auparavant voient assez rapidement leur rôle de centres diffuseurs changer de configuration, voire d'importance.

\section{BIBLIOGRAPHIE}

Arce Vargas, Rejane M., 2011, Designação e dessignificação: a filiação de sentidos na fraseologia contemporânea, Santa Maria, Brésil, Université Fédérale de Santa Maria/PPGL, [En ligne], http:// cascavel.cpd.ufsm.br/tede/tde_busca/arquivo.php?codArquivo=3818 (Thèse de doctorat de Lettres).

Casilli, Antonio, 2010, Les Liaisons numériques. Vers une nouvelle sociabilité ?, Paris, Seuil.

Castells, Manuel, 2012, Redes de indignación y esperanza. Los movimientos sociales en la era de internet, Trad. esp. de María Hernández, Madrid, Alianza Editorial.

Courtine, Jean-Jacques, 1981, « Quelques problèmes théoriques et méthodologiques en analyse du discours, à propos du discours communiste adressé aux chrétiens », Langages, $\mathrm{n}^{\circ}$ 62, p. 9-128.

Dias, Cristiane (dir.), 2011, E-urbano. Sentidos públicos do espaço urbano/digital, São Paulo, Unicamp, Labeurb, [En ligne], http://www.labeurb.unicamp.br/livroEurbano/index.htm (e-book).

-, 2008, Da Corpografia: ensaio sobre a língua/escrita na materialidade digital, Santa Maria, UFSM / PPGL, [En ligne], http://coral.ufsm.br/mletras/images/Cogitare07.pdf.

Fenoglio, Irène, 1997, « La notion d'événement d'énonciation : le "lapsus" comme une donnée d'articulation entre discours et parole ", Langage et société, nº 80, p. 39-71.

Guilhaumou, Jacques et Maldidier, Denise, 1986, « Effets de l'archive. L'analyse de discours du côté de l'histoire ", Langages, $\mathrm{n}^{\circ}$ 81, p. 43-56.

-, 1986a, « De l'énonciation à l'événement discursif en analyse de discours », dans Histoire

Épistémologie Langage, t. vIII, fasc. 2, p. 233-242.

Indursky, Freda, 2002, « Do desdobramento à fragmentação do sujeito em análise do discurso », dans Sínteses 2, [CD-ROM], Porto Alegre, ANPOLL / UFRGS. 
-, 2005, « Formação discursiva: ela ainda merece que lutemos por ela por ela? », dans Seminário de Estudos em Análise do Discurso. 0 campo da análise do discurso no Brasil: mapeando conceitos, confrontando limites, 2, Porto Alegre, UFRGS, [En ligne], http://www.ufrgs.br/analisedodiscurso/ anaisdosead/2SEAD/SIMPOSIOS/FredaIndursky.pdf, consulté le 5 juillet 2013.

Orlandi, Eni, 2010, « A contrapelo. Incursão teórica na tecnologia: discurso eletrônico, escola, cidade », Rua, n 16, vol. 2, [En ligne], http://www.labeurb.unicamp.br/rua/pages/ pdf/16-2/1-16-2.pdf.

Paveau, Marie-Anne, 2006, Les Prédiscours. Sens, mémoire, cognition, Paris, Presses Sorbonne Nouvelle.

-, 2011, « \#EvenementDiscursif \#Sidibouzid \#fb », La pensée du discours, [Carnet de recherche], http://penseedudiscours.hypotheses.org/3222, consulté le 15 mai 2012.

-, 2012a, « Les amitiés numériques. Formes technolangagières et polysémie relationnelle », La pensée du discours, [Carnet de recherche], http://penseedudiscours.hypotheses.org/8334, consulté le 15 juin 2013.

-, 2012b « Ah. Un "vrai” livre numérique? », Technologies discursives, 28 juillet 2012, http:// technodiscours.hypotheses.org/189, consulté le 23 juin 2013.

-, 2012c, « Activités langagières et technologie discursive. L'exemple de Twitter », La pensée du discours, [Carnet de recherche], http://penseedudiscours.hypotheses.org/8338, consulté le 14 mai 2012.

,- 2013a, « Genre de discours et technologie discursive. Tweet, twittécriture et twittérature », Pratiques, $\mathrm{n}^{\circ} 157-158$, p. 7-30. (Les pages indiquées dans les citations sont de la version auteur : $<$ hal-00824817>.)

-, 2013b, « Technodiscursivités natives sur Twitter. Une écologie du discours numérique », Epistémé, $\mathrm{n}^{\circ}$ 9, p. 139-176. (Les pages indiquées dans les citations sont de la version auteur : $<$ hal-00859064>.)

Pêcheux, Michel, [1983] 1990, «Le discours : structure ou événement? », dans L'Inquiétude du discours, textes de Michel Pêcheux choisis et présentés par Denise Maldidier, Paris, Éditions des Cendres, p. 303-323.

Pêcheux, Michel et Fuchs, Catherine, 1975, « Mises au point et perspectives à propos de l'analyse automatique du discours ", Langages, $n^{\circ} 37$, p. 7-80.

Rancière, Jacques, 1995, La Mésentente. Politique et philosophie, Paris, Éditions Galilée.

Serres, Michel, 2012, Petite Poucette, Paris, Le Pommier.

\section{NOTES}

1. Traduction : «Père, éloigne de moi, ce calice. » La composition musicale brésilienne "Cálice » joue avec l'homophonie des mots cálice (nom) et cale-se (verbe qui signifie «se taire», ici à l'impératif : « taisez-vous »). Cette chanson célèbre a été composée pendant la dictature militaire et abordait ce qui était interdit à ce moment historique, par le biais de la transgression discursive, de la polysémie, de la polyréférentialité, tout en désacralisant le discours biblique. La chanson a été censurée pendant plusieurs années et fait partie de l'histoire des interdits brésiliens qui sont remémorés et actualisés lors d'événements polémiques comme les manifestations de juin 2013. On peut la lire et l'écouter sur: http://letras.mus.br/miltonnascimento/112696/. 
2. Voir http://www.rfi.fr/ameriques/20130618-vaste-mouvement-protestation-bresil.

3. Disponible sur la page : http://tempsreel.nouvelobs.com/economie/20130618.OBS3696/bresille-miracle-economique-perd-de-sa-superbe.html.

4. Voir http://www1.folha.uol.com.br/cotidiano/2013/06/1296886-em-protesto-de-sp-maiorianao-tem-partido-diz-datafolha.shtml.

5. Voir http://www.brasildefato.com.br/node/13263.

6. La notion de matérialité discursive est considérée ici dans la relation entre langue et processus discursif, conformément à la pensée de Pêcheux et Fuchs (1975: 16) : «Les processus discursifs étant à la source de la production des effets de sens, la langue constitue le lieu matériel où se réalisent ces effets de sens " (nous soulignons).

7. Selon Courtine (1981) la mémoire discursive recouvre l'ensemble des dires possibles, puisqu'elle relève de l'existence historique des énoncés, des discours au sein des pratiques discursives, en permettant qu'ils soient répétés, reformulés, etc. La notion reste attachée au concept de formation discursive (en ce qui concerne le concept de base chez Foucault 1969), emprunté et développé par Pêcheux et Fuchs (1975). D’une façon assez minimaliste, on pourrait dire que la mémoire discursive comporte l'ensemble des dires constitués historiquement comme ce qui peut et doit être dit dans une conjoncture socio-historique donnée, mais cela pose certains problèmes concernant sa matérialité (ceci sans approfondir la question de l'hétérogénéité et des reformulations que ce concept a connues au long de ces trente dernières années). Dans les limites de ce texte, nous concevons la mémoire discursive comme l'inscription des dires dans un réseau dynamique, à la fois synchronique et diachronique.

8. Conformément aux déclarations de Bruno Torturra, l'un des théoriciens de Média Ninja ; voir l'entretien sur: http://www.youtube.com/watch?v=M4VmRH55JMo.

9. Voir ce texte du 21 juillet 2013, « Ninja, le média social des manifestations au Brésil »: http:// america-latina.blog.lemonde.fr/2013/07/21/ninja-le-media-social-des-manifestations-au-bresil/. 10. Paveau (2012a) donne comme exemples de gestes technolangagiers ou technoscripturaux : les boutons avec ou sans texte comme «like» de FB, les boutons de partage, de demande d'amitié ( ajouter»); l'insertion de liens automatiques sur les réseaux comme FB; le partage de lien comme forme de discours rapporté; le commentaire comme forme spécifique d'interaction verbale, conversationnelle; le mot cliquable comme le hashtag.

11. Au sens de Courtine (1981), nous comprenons cela comme l'ensemble constitué de formulations préalables et repérables au niveau de l'intradiscours et qui sont, dans ce cas, mises en cause en tant que formulations de référence d'un domaine $\mathrm{X}$.

12. Rancière conçoit la mésentente en tant qu'un «type déterminé de situation de parole : celle où l'un des interlocuteurs à la fois entend et n'entend pas ce que dit l'autre. La mésentente n'est pas le conflit entre celui qui dit blanc et celui qui dit noir. Elle est le conflit entre celui qui dit blanc et celui qui dit blanc mais n'entend point la même chose ou n'entend point que l'autre dit la même chose sous le nom de la blancheur. [...] Les cas de mésentente sont ceux où la dispute sur ce que parler veut dire constitue la rationalité même de la situation de parole » (Rancière 1995 : 12-13).

13. Selon Paveau (2012c) ce sont des univers de discours en ligne pleinement intégrés à nos modalités de parole et d'échange quotidiens, lesquelles se trouvent augmentées par la technologie, selon le principe de la réalité augmentée.

14. Voir Paveau (2011).

15. Michel Serres (2012) a donné le nom de Petite Poucette à la génération née au sein des nouvelles technologies, laquelle, selon lui, a un rôle fondamental dans les sociétés contemporaines, celui de réinventer les formes d'être, de connaître, de vivre ensemble, de partager les savoirs. À notre sens, les événements de juin 2013 au Brésil témoignent du cadre dessiné par l'auteur. 
16. Le journal Folha de São Paulo affirme que la plupart des manifestants a entre 26 et 35 ans et que $81 \%$ ont pris connaissance des manifestations sur Facebook et $85 \%$ sur Internet. Voir http:// www1.folha.uol.com.br/cotidiano/2013/06/1296886-em-protesto-de-sp-maioria-nao-tempartido-diz-datafolha.shtml.

17. Voir l'article «Au Brésil, la génération Facebook fait sa révolution », RFI, http://www.rfi.fr/ ameriques/20130620-brazil-facebook-pele-youtube-anonymous-dilma-rousseff.

18. Voir l'image, probablement un photomontage, où le fondateur de Facebook apparaît et est présenté comme soutenant le mouvement sur : http://glamurama.uol.com.br/mark-zuckerbergda-seu-apoio-as-manifestacoes-brasileiras-vem-ver/, consulté le 20 juin 2013.

19. Voir les paroles de la chanson sur http://letras.mus.br/thiago-correa/brasil-em-cartaz/.

20. Voir la vidéo de la chanson sur: https://www.youtube.com/watch? feature=player_embedded\&v=PJ6NnGEiucM.

21. Le mashup est un style musical proche du pot-pourri qui associe différents éléments de plusieurs chansons dans un seul morceau. Il est lié aux univers numériques.

22. Voir un storify de Thomas Bourdeau à ce propos sur : http://storify.com/tbourdeau/bresilbrazil-manifestation-sao-paulo-pele, consulté le 22 juin 2013.

23. «Mouvement protestataire de 2013 au Brésil», Wikipedia, http://fr.wikipedia.org/wiki/ Mouvement_protestataire_de_2013_au_Brésil, consulté le 24 juin 2013.

24. Avertissement en haut de la page: "Cet article concerne un événement en cours. Ces informations peuvent manquer de recul et changer à mesure que l'événement progresse. Le titre lui-même peut être provisoire. N'hésitez pas à l'améliorer en veillant à citer vos sources. Dernière modification de cette page le 25 juin 2013 à $11: 25$. »

25. Storify est une plate-forme qui permet d'élaborer un story telling à partir de liens FB, YouTube, Twitter, Flickr, Instagram, etc. tout en conservant la nature, l'environnement multisémiotique de ces sources (son, image, vidéo), voir http://storify.com.

26. Voir à ce sujet : «La "révolte du vinaigre" secoue le Brésil », Global voices, [En ligne], http:// fr.globalvoicesonline.org/2013/06/16/148389/, consulté le 24 juin 2013; ainsi que ce texte à propos d'un journaliste arrêté pour porter du vinaigre, sur: http://extra.globo.com/noticias/ brasil/reporter-preso-em-sao-paulo-por-portar-vinagre-durante-manifestacoes-8689239.html.

27. http://blogs.mediapart.fr/blog/ana-guerra/180613/le-bresil-deja-gagne.

28. Mouvement qui lutte pour la qualité et la gratuité des transports en commun à São Paulo (ville), voir http://saopaulo.mpl.org.br/apresentacao/.

29. Vidéo sur YouTube : « Gad ElMaleh smileys sms msn facebook», https://www.youtube.com/ watch?v=cugImEJoGyg.

30. Rappelons que l'action d'ignorer est réalisée par l'intermédiaire d'un bouton "ignorer»; dernièrement, pour répondre à une demande d'amitié, ont été mis en place deux boutons: « confirmer » ou « plus tard».

31. Paveau définit le genre discursif comme « un ensemble de cadres collectifs pré-, extra- et intra-discursifs, constitutifs de l'élaboration-interprétation des énoncés » et les énoncés comme des «productions matérielles langagières en situation » (2013a:5).

32. Voir ici même l'article de Cristiane Dias.

33. Voir à propos de la multimodalité le texte sur le site Global Voices, réseau mondial de blogueurs http://fr.globalvoicesonline.org/2013/06/16/148389/.

34. Voir http://tempsreel.nouvelobs.com/economie/20130618.0BS3696/bresil-le-miracleeconomique-perd-de-sa-superbe.html\#reagir.

35. «Évidemment, la technologie ne détermine aucun mouvement ni aucun comportement social. Mais Internet et les réseaux de téléphonie mobile ne sont pas de simples outils, sinon des formes d'organisation, des expressions culturelles et des plates-formes spécifiques d'autonomie politique » (nous traduisons). 
36. Voir à propos du contexte brésilien, en particulier concernant le rapport urbain-digital, les travaux de Dias $(2008,2011)$.

37. «Cet hybride du cyberespace et de l'espace urbain est un troisième espace que j'appelle l'espace de l'autonomie » (nous traduisons).

38. Voir http://www.brasildefato.com.br/node/13263.

39. Les traductions ont été réalisées dans le but d'être le plus proche possible de la langue source, tout en faisant des adaptations au profit de la cohérence.

40. Voir http://www1.folha.uol.com.br/cotidiano/2013/06/1296886-em-protesto-de-sp-maiorianao-tem-partido-diz-datafolha.shtml.

41. Voir http://tempsreel.nouvelobs.com/economie/20130618.0BS3696/bresil-le-miracleeconomique-perd-de-sa-superbe.html.

42. Voir http://www.rfi.fr/ameriques/20130618-vaste-mouvement-protestation-bresil/.

43. Voir http://tempsreel.nouvelobs.com/economie/20130618.0BS3696/bresil-le-miracleeconomique-perd-de-sa-superbe.html.

44. Voir http://www.brasildefato.com.br/node/13263.

45. Voir http://www1.folha.uol.com.br/cotidiano/2013/06/1296886-em-protesto-de-sp-maiorianao-tem-partido-diz-datafolha.shtml.

46. Selon le site du journal, qui s'appuie sur les données de l'IVC (Institut vérificateur de circulation) d'octobre 2012, il s'agit de l'un des médias les plus lus au Brésil, voir http:// www1.folha.uol.com.br/institucional/circulacao.shtml.

47. Vérifié le 22 juin 2013 à 14 h 49.

48. Voir la politique de commentaires du site: http://www1.folha.uol.com.br/paineldoleitor/ comentarios/.

49. Nous avons travaillé cette notion dans notre thèse (Arce Vargas 2011), également dans le contexte des discours multimodaux, notamment numériques.

50. Voir http://fr.globalvoicesonline.org/2013/06/16/148389/.

51. Voir https://storify.com/tbourdeau/bresil-manifestations-salad-vinaigre.

52. Voir https://storify.com/tbourdeau/bresil-brazil-manifestation-sao-paulo-pele.

53. Voir http://blogs.mediapart.fr/blog/ana-guerra/180613/le-bresil-deja-gagne.

\section{RÉSUMÉS}

Nous proposons une réflexion autour des outils numériques qui offrent aux lecteurs des possibilités de partager des informations tout en créant des nouveaux espaces de discursivisation. Pour illustrer notre pensée, nous avons choisi des matérialités discursives multisémiotiques collectées sur les sites web Le Nouvel Observateur, Radio France Internationale, Folha de São Paulo et Brasil de Fato. Le but est d'établir un contraste entre certains aspects constitutifs des textualités des médias dits traditionnels et des post-médias, soit les nouveaux contextes de profusion de discours ouverts par les réseaux sociaux numériques (RSN), en se focalisant sur différentes manières de diffuser et de participer à la fabrication d'un événement discursif. Ainsi, nous discuterons des dimensions que ces sociabilités numériques apportent à la notion d'événement, dans un univers où la forme linguistique s'associe à de nombreuses expressions intraduisibles par l'écriture qui, pourtant, contribuent à la construction du sens de cette même écriture. 
We propose a reflection on the digital tools that give readers opportunities to share information, thus creating new spaces for discursivization. To illustrate our thinking, we chose multi-semiotic discursive materialities collected on the sites Le Nouvel Observateur, Radio France Internationale, Folha de São Paulo and Brasil de Fato. The goal is to establish a contrast between certain constituent aspects of textualities of the so-called traditional media and post-media, that is, the new contexts for profusion of discourses opened by digital social networks (DSN) focusing on the different ways to disseminate and participate in the making of a discursive event. Thus, we discuss the dimensions these digital sociabilities bring to the notion of event in a world where the linguistic form is associated with numerous untranslatable expressions by writing, which, however, contribute to the construction of sense of such writing.

\section{INDEX}

Keywords : enunciative event, discursive event, digital links, techno-discourse, post-media Mots-clés : événement énonciatif, événement discursif, liaisons numériques, technodiscours, post-médias

\section{AUTEUR}

\section{REJANE ARCE}

Laboratoire Corpus, UFSM, Brésil ; Pléiade (EA 7338), Université Paris 13, Sorbonne Paris Cité, France 